\title{
Transmission and Detection of Electromagnetic Waves for Gesture Recognition
}

\author{
Sandeep Kaur and Bhavneesh Sohal
}

Computer Science and Engineering, Lovely Professional University, Jalandhar-Delhi, G.T. Road, National Highway 1, Phagwara - 144411, Punjab, India; Sandeep.16827@lpu.co.in, sohal.16042@1pu.co.in

\begin{abstract}
Hand gesture technology makes users' lives simpler, achieving "hands free" interaction through eliminating the need to hold or press the device. Since the need of human computer interaction has increased to a great-extent, such a technique needs to be devised that do not depend on Vision or Wearable sensors approach. This paper is a sincere attempt to recognize gestures using EM field detection approach. This requires only an EM field transmitter and a detector (receiver). The novelty of this approach is, it not only reduces extra cost for wearable or vision based sensors but would also account for a wide range of coverage area. In addition to this, method to recognize gestures through EM Waves with the help of a laser light is also practically implemented. The demonstration of this method is illustrated with the help of Macromedia Flash Player. This theory will certainly reduce the cost factor and add to the simplicity of the current human computer interactions.
\end{abstract}

Keywords: Detector, Doppler Shift, Electromagnetic, Flux Points, Gesture Recognition, Light Sensor

\section{Introduction}

HCI (Human Computer Interaction) is defined as the interaction between humans and computers. As it involves both the machine and computer interaction hence it requires support from both the ends. On the system/computer end, the following points are involved: Computer Graphics, Operating System and programming languages and on the human end: communication theory, graphic and industrial design disciplines, linguistics, social sciences, cognitive psychology are associated. HCI basically includes gesture recognition, speech recognition and eye tracking.

In this paper, recognition is performed using gestures. Gesture Recognition is defined as the technique of recognizing and interpreting gestures. The gestures mainly originate from Arm or Hand, Neck or Head and Body. However, the techniques being developed nowadays mainly focus on the Face and Hand recognition. Gesture recognition has been found useful in bridging the gap between user and machines and has significantly devel- oped GUI (graphical user interface) features. Gesture Recognition is used in recognizing sign language, pointing for directional purposes, facial control, alternative computer interfaces, controlling through remotes. There has been significant development in Gesture Recognition over the past one decade and many new technologies have been devised. Data Gloves or video cameras are used extensively for the same. Due to their complexity and expense, they are avoided to be brought in frequent use. Recent study on gesture recognition using wireless signals involving Doppler shifts has proved that still there is much scope left in development of techniques involving EM (Electromagnetic) waves.

This paper works on gesture recognition system that uses wireless signals to enable sensing and recognition of human gestures. Since wireless signals do not require line-of-sight and can traverse through walls, gesture recognition can be achieved without requiring human body sensing devices. This can be achieved by looking at the Doppler shifts and multi-path distortions that occur with these wireless signals from human motion in the envi-

*Author for correspondence 
ronment. Doppler shift occurs whenever a wave source moves relative to the observer. In the context of wireless signals, if we consider the multi-path reflections from the human body as waves from a source, then a human performing a gesture, results in a pattern of Doppler shifts at the wireless receiver.

\section{State-of-the-Art}

This section discusses the work done by the earlier researchers.

${ }_{1}^{1}$ Presented work on Homodyne Sensors that worked same as EM wave interferometer by comparing the reflection of a transmitted wave against a local wave. When reflecting surface of the system moves, the phase of the reflected wave varies with respect to the stationary local wave. Hence, the detection system measures position changes versus time, within the specific frequency range.

2Proposed a technology that was significant enough to start a new field of research since it introduced gesture recognition by EM (Electromagnetic Waves). Author introduced a new sensor (Homodyne sensor) that worked as a wave interferometer by comparing the reflection of a transmitted wave against a local (phase reference) wave. This sensor was found effective in capturing the gestures performed on a larger extent.

${ }^{3}$ Defined advancement in gesture recognition with the help of MEMS accelerometer. Accelerometers are the instruments that can detect the acceleration by which a hand moves. Eight gesture codes were extracted and were matched with the values stored in database. The model was efficient in recognizing seven hand gestures.

4Presented a work that focused on the Scale Variance and introduced SIFTS (Scale Invariant Feature Transform) Algorithm. Scale Invariance was done with the help of DOG (Difference of Gaussian) i.e. the difference between two Gaussian Surfaces is calculated. The algorithm discussed here states that whenever any image is encountered, its key points are stored in the database and the respective DOG values are also determined. Next time whenever a match had to be made, the key points are compared with the earlier stored values and accordingly the match is made. MATLAB tool is used for successful matching of the data values.

5Provided work on static gestures combining with graphs that mainly focused on the features that are extracted in the process of gesture recognition. SGONG algorithm was used to create an approximation of the hand topology. Particular methods were developed to detect and process canny edges.

${ }^{6}$ Presented work on a new technique that identified low level hand movement by classifying the single channel sEMG. Single channel sEMG analysis was preferred over multi-channel due to its simplicity, computational cost and efficiency. Author defined the Wavelet transformation and artificial neural network (ANN) classifier to classify and analyze the sEMG signal in a better way.

${ }^{7}$ Defined a vision based recognized gestures to control multimedia applications (like Windows Media Player, Windows Picture Manager, VLC Player etc.) running on computer using different gestural commands. Author recognized the static hand gesture images (i.e. frames) based on shapes and orientations of hand which were extracted from input video stream recorded in stable lighting and simple background conditions.

${ }^{8}$ Provided work on arm gesture using artificial neural network (ANN) training with a Learning Vector Quantization (LVQ) algorithm. Author proposed a cost effective low power wearable wrist band to control the locomotion of robot using static gesture from hand which leads to the advance concept of unmanned vehicle.

${ }^{9}$ Developed a Face Recognition model as a replacement for default lock screen based on Viola and Jones method for detection of face. Author used a single web camera as input device to recognize gestures of hand. Some of these gestures include controlling the mouse cursor, clicking actions and few shortcuts for opening specific applications. Author implemented PCA (Principal Component Analysis) for recognition and identification of algorithms.

${ }^{10}$ Provided work on different sets of hand gestures using artificial neural network (ANN) and feature extraction techniques. Author proposed an algorithm that helped in recognizing the different signs and converting gestures into voice. Author recognized the hand gestures from the extracted frames by using background subtraction techniques.

${ }^{11}$ Presented a comparative study on various methodologies for facial age estimation. Author discussed various tools used for feature extraction and normalization. Author implemented LBP, DCT Mod2 and HOG and compared against each other.

\section{Conceptual Design}

EM fields are found everywhere, some spectrums are visible to naked eye and some are not. If EM waves are 
distorted at particular points, they would generate a pattern. So, successful recognition and tracing of this pattern can be used for gesture recognition. Introducing an EM field theory for detecting gestures not only reduces extra cost for wearable or vision based sensors but would also account for a wide range of coverage area. In this paper, proposed theory works with the transmission of EM wave and its detection at various points to find appropriate gestures. This approach is useful in the following ways:

- This technique is cheaper than other approaches introduced earlier.

- Since EM fields are found everywhere, the range would be comparatively wider.

- The devices and tools required for the implementation are simple.

- Unlike wearable sensors, the apparatus involved in this approach doesn't cause any harm to human body.

Since the need of HCI has increased to a greater extent, such a technique needs to be devised that does not depend on Vision or Wearable sensors approach as they had the following disadvantages.

\section{Disadvantages of Wearable Sensors}

- Wearable sensors need to be wore in hand or arm for Gesture Recognition.

- Wearable sensors like accelerometers are expensive.

\section{Disadvantages of Vision Based Sensors}

- They are not very accurate.

- Environmental factors like brightness or same colour contrast may hinder the gesture recognition.

\section{A. Objective}

An alternate and efficient approach to the existing techniques is EM field detection approach. This requires only an EM field transmitter and a detector (receiver). Our aim here is to refine this approach and examine its testing in practical. The generated EM wave can be detected using a detector. The apparatus used for the same has an energy source that will travel in the circuit and will generate an EM wave that will be transmitted through the transmission line. Our proposed theory is that, if the apparatus is attached with the human hand and the gestures are performed, the detector will detect the EM waves at various gesture points forming a grid, then the proper gesture can be extracted out of it. Electromagnetic waves are of several spectrums classified on the basis of frequencies. The chart of electromagnetic spectrum is given in Figure 1. Different spectrums can be used for gesture recognition. Electromagnetic waves or EM waves is simply a wave having both electric and magnetic fields. EM waves are present in atmosphere in many forms, some are visible to naked eye and some are not. Now discussing how the EM waves are produced. When the current is made to flow outside the conductor (mainly in rotation) then EM waves are produced that can be plotted on a machine when interfaced with computer system.

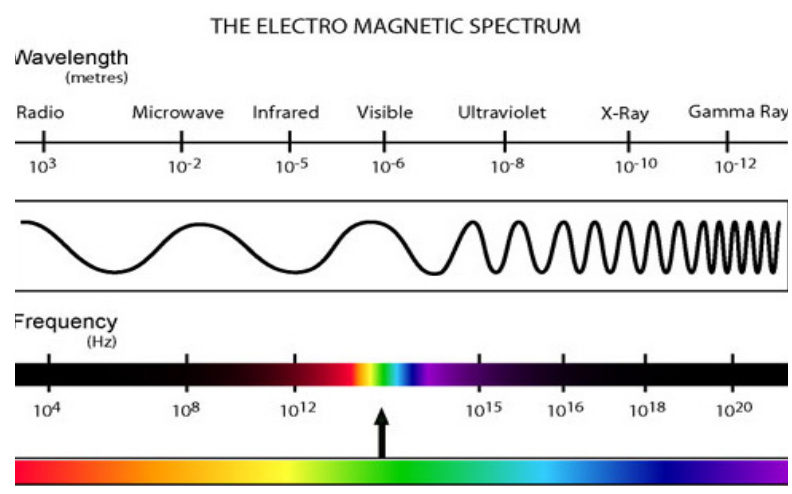

Figure 1. EM Wave Spectrum.

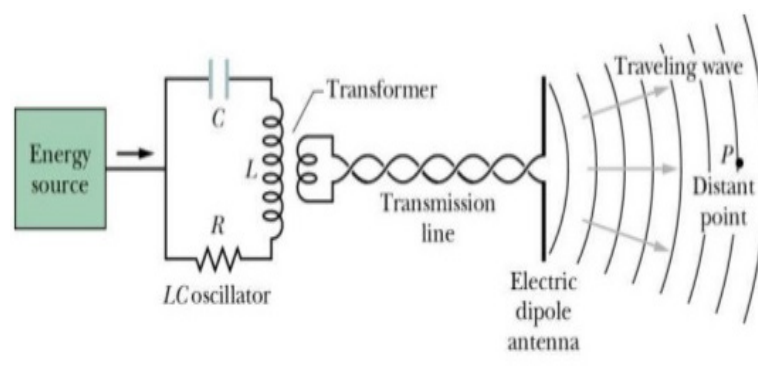

Figure 2. EM Wave Generator.

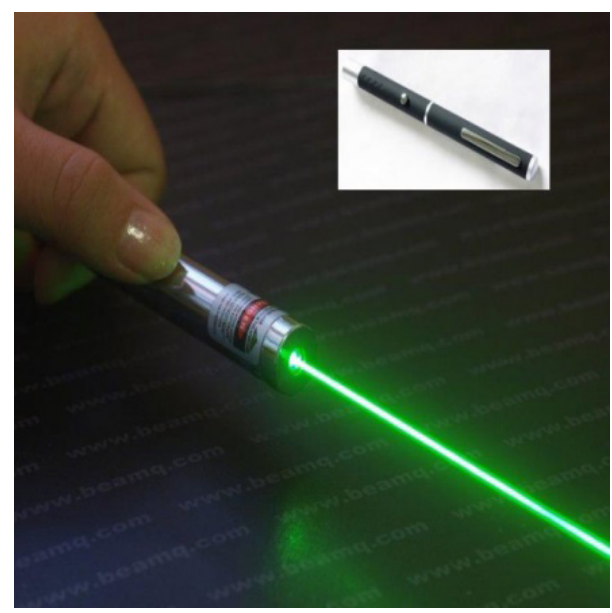

Figure 3. Beam transmitted by laser light device. 
A simple apparatus to generate an EM wave is shown in Figure 2. LC oscillator produces a sinusoidal current in the antenna, which generate the wave. $\mathrm{P}$ is a distant point at which a detector can monitor the wave travelling past it.

Second approach to Gesture Recognition through EM Waves is with the help of a laser light. The light emitted by a laser light is also a form of EM wave. This light can be sensed using light sensor which precisely estimates the location of the light and the intensity of the light beam. An example of laser light and light sensor is illustrated in Figure 3 and Figure 4.

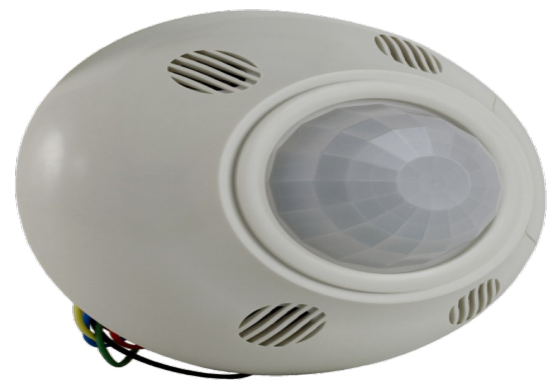

Figure 4. Light Sensor.

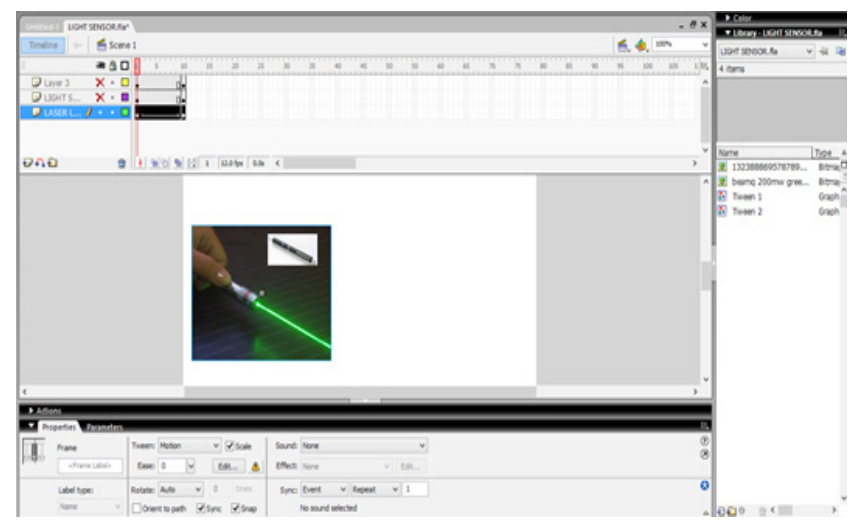

Figure 5. Layer 1 (Laser Light).

\section{Methodology and Implementation}

\section{B. Research Methodology}

The demonstration of the methods discussed above is illustrated with the help of Macromedia Flash Player. In the first demonstration, the laser light is intended to be targeted towards the light sensor. The light sensor is a device that tells about the intensity of the light and the estimated location of the light. Now the intensity being constant in this case, the changing location would help to predict the gesture. Once the light sensor senses the light, the noise filter would filter the extra light of the surroundings. In the next step, the connection is made to the PC where the gestures are interpreted and further matched with the set of gestures. The steps of recognition are displayed in subsequent figures. First layer is shown in Figure 5. Here, only the laser beam appears, keeping the second and third layer as constant.

Second layer is shown in Figure 6. In this layer, only the sensor is placed in the layer, keeping the other layers as constant.

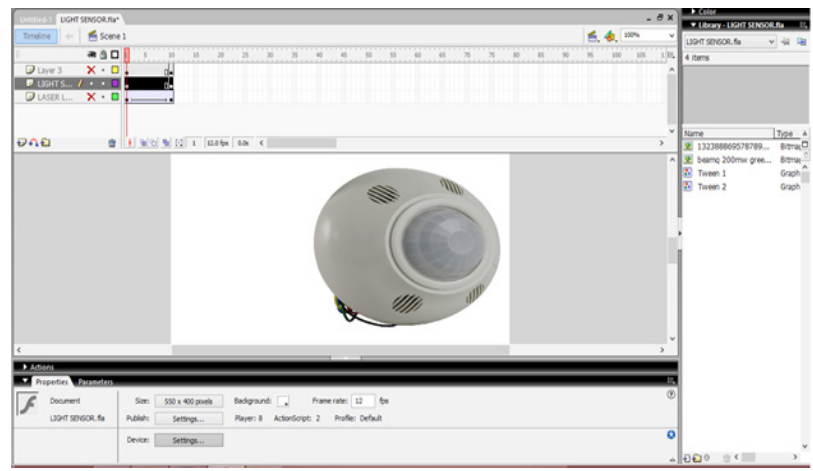

Figure 6. Layer 2 (Light Sensor).

Third layer is shown in Figure 7. In this layer only the text portion appears keeping the other layers as constant.

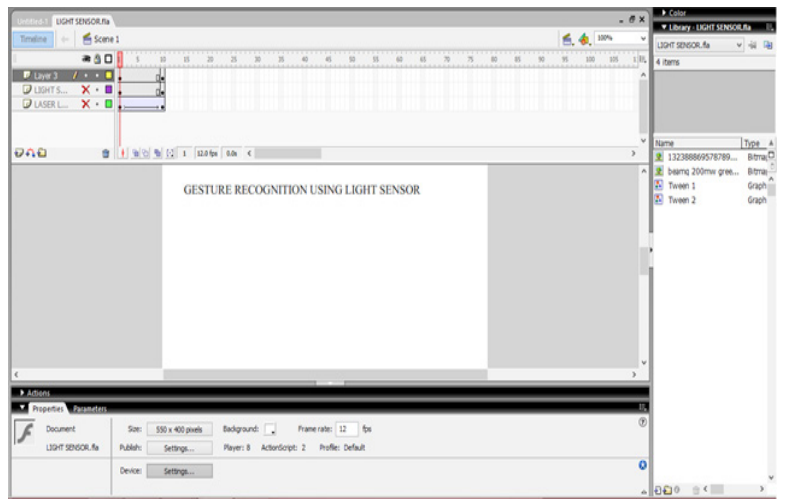

Figure 7. Layer 3.

Figure 8 shows the final animation.

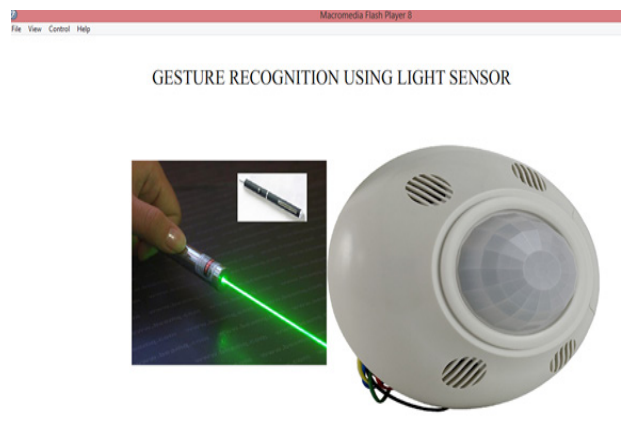

Figure 8. Final Working. 
Figure 9 shows scenario of interfacing light sensor with computer system.

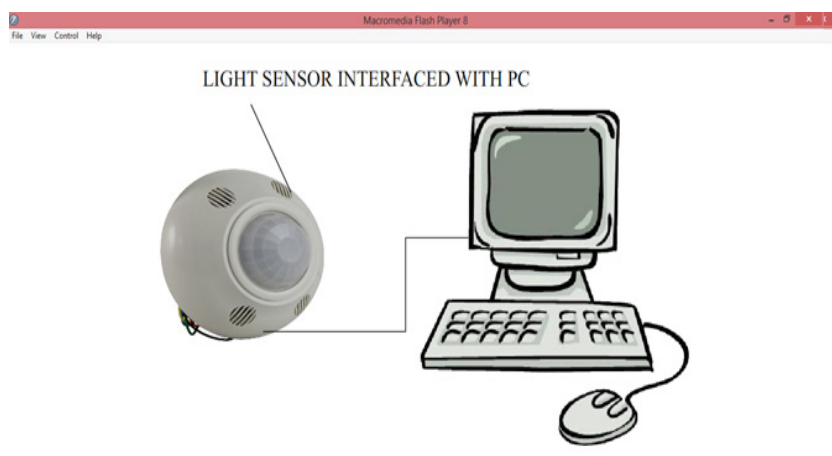

Figure 9. Sensor interfaced with PC.

In second demonstration, the EM wave generator is attached to hand. As the hand moves, the waves generated ${ }^{*}$ from various points consequently form the flux points which would therefore help in tracing the trajectory of the gestures. The only difference is that the EM wave generator is attached to the hand and the gestures are performed. Figure 10 shows EM Wave receiver.

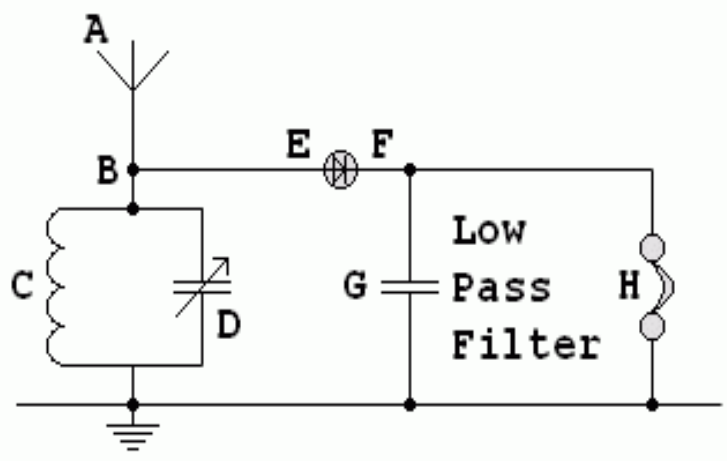

Figure 10. EM Wave Receiver.

\section{Conclusion}

In the scenario of advancements in technology, there is a need to develop HCI to a much greater extent.

Till now many techniques have been employed for gesture recognition. Only need is to device such a technique that not only gives accurate results but is also cheap and can be easily brought to use. In this paper, a novel tech- nique is proposed to interact with computer over a wider range. Neglecting the noise factor, there will be interruptions in magnetic field lines that would be detected by a detector. This theory will certainly reduce the cost factor and add to the simplicity of the current human computer interactions.

\section{References}

1. Holzrichter JF, Ng LC and Livermore L. Speech Articulator and User Gesture Measurements Using Micropower, Interferometric EM-Sensors. Institute of Electrical and Electronics Engineers. 2001 May; p. 21-3.

2. Pu Q, Gupta S, Gollakota S and Patel S. Whole-home gesture recognition using wireless signals. 2013 July; p. 485-86.

3. Xu R, Zhou S and Li W. MEMS Accelerometer Based Non-Specific-User Hand Gesture Recognition. Institute of Electrical and Electronics Engineers Sensors Journal. 2012 May; 12(5):1166-73.

4. Nachamai M. Alphabet Recognition of American sign language: A hand gesture recognition approach using SIFT algorithm. International Journal of Artificial Intelligence \& Applications. 2013 January; 4(1):105-15.

5. Avraam M. Static Gesture Recognition Combining Graph and Appearance Features. International Journal of Advanced Research in Artificial Intelligence. 2014 May; $3(2): 1-4$.

6. Mane SM, Kambli RA, Kazi FS, Singh NM. Hand Motion Recognition From Single Channel Surface EMG Using Wavelet \& Artificial Neural Network. Procedia Computer Science. 2015 April; 49(2015):58-65.

7. Sharma RP and Verma GK. Human Computer Interaction using Hand Gesture. Procedia Computer Science. 2015 June; 54(2015):721-27.

8. Chakravarthi MK, Tiwari RK and Handa S. Accelerometer Based Static Gesture Recognition and Mobile Monitoring System Using Neural Networks. Procedia Computer Science. 2015 October; 70(2015):683-87.

9. Lalithamani N. Gesture Control Using Single Camera for PC. Procedia Computer Science. 2016 Feburary; 78(2016):146-52.

10. Kanchana Devi P, Raji A and Srinivasan G. Gesture Recognition for Physically Challenged. Indian Journal of Science and Technology. 2016 April; 9(16):1-5.

11. Deepa A, Sasipraba T. Challenging Aspects for Facial Feature Extraction and Age Estimation. Indian Journal of Science and Technology. 2016 January; 9(4):1-6. 